$\begin{array}{ll}\text { Research Square } & \begin{array}{l}\text { Preprints are preliminary reports that have not undergone peer review. } \\ \text { They should not be considered conclusive, used to inform clinical practice, } \\ \text { or referenced by the media as validated information. }\end{array}\end{array}$

\title{
Comparing ERAS-Outpatient Versus Standard-Inpatient Hip and Knee Replacements: A Mixed Methods Study Exploring the Experience of Patients Who Underwent Both
}

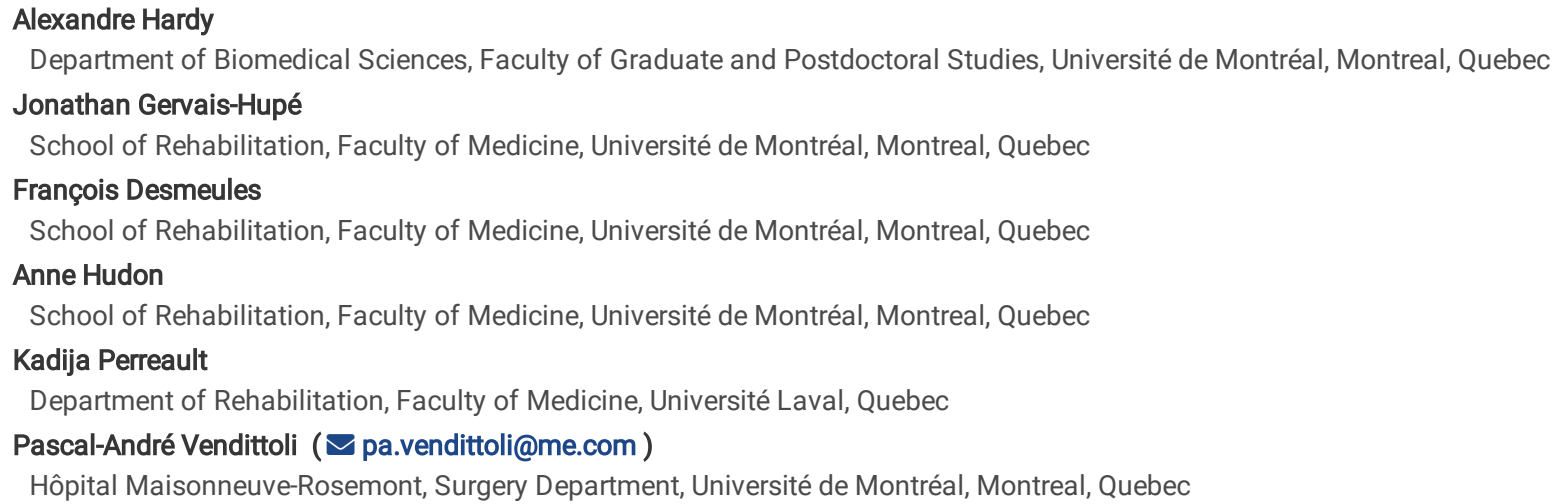

Keywords: Arthroplasty, Replacement, Knee, Arthroplasty, Replacement, Hip, Enhanced recovery after surgery, Fast-track, Outpatient, Patient Experience, Patient Outcome Assessment, Mixed methods research

Posted Date: July 6th, 2021

DOl: https://doi.org/10.21203/rs.3.rs-589386/v1

License: @ (i) This work is licensed under a Creative Commons Attribution 4.0 International License. Read Full License

Version of Record: A version of this preprint was published at BMC Musculoskeletal Disorders on November 23rd, 2021. See the published version at https://doi.org/10.1186/s12891-021-04847-9. 


\section{Abstract \\ BACKGROUND}

Optimizing patients' total joint arthroplasty (TJA) experience is as crucial for providing high quality care as improving safety and clinical effectiveness. Yet, little evidence is available on patient experience in standard-inpatient and enhanced recovery after surgery (ERAS)-outpatient programs. Therefore, this study aimed to gain a more in-depth understanding of the patient experience by exploring the patient experience of ERAS-outpatient programs compared to standard-inpatient programs, identifying elements that could optimize patients' experience and determining how it is associated with patient characteristics, clinical outcomes and care components satisfaction.

\section{METHODS}

We conducted a convergent mixed methods study of 48 consecutive patients who experienced both standard-inpatient and ERAS-outpatient TJA contralaterally. A reflective thematic analysis was conducted based on data collected via a questionnaire. Bivariate correlations between the patient experience and patients' characteristics, clinical outcomes and care components satisfaction were performed. Then, the quantitative and qualitative data were integrated together.

\section{RESULTS}

The theme Support makes the difference-for better and for worse was identified in both programs and throughout the entire TJA care episode. Patients identified 3 main themes distinguishing the ERAS-outpatient program from their standard-inpatient experience: 1) Minimizing inconvenience, 2) Home sweet home and 3) Returning to normal function and activities. Providing more preoperative information and postoperative rehabilitation sessions (if needed) and ensuring better coherence of care between orthopaedic and homecare teams could further optimize the patient experience. Weak to moderate positive and statistically significant correlations were found between patients' TJA experience and satisfaction with pain management, hospital stay, postoperative recovery, homecare and overall results $\left(r_{s}=+[0.36-0.66]\right.$, $p$-value $\left.<0.01\right)$

\section{CONCLUSION}

Whatever the perioperative program, the key to improving patients' TJA experience lies in improving support throughout the care episode. Compared to standard-inpatient care, the ERAS-outpatient program improves patients' experience by providing dedicated support in post-operative care, reducing postoperative inconvenience, optimizing pain management, returning home sooner, and recovering and regaining function sooner. Patients' TJA experience could further be enhanced by optimizing the information provided to the patient, the rehabilitation program and the coherence between care teams.

\section{Introduction}

Fast-track protocols represent a potential solution to the challenges posed by the expected increase in demand for total joint arthroplasty (TJA) over the coming decades and wait times exacerbated by the COVID pandemic. ${ }^{1,2,3}$ The shift from inpatient to outpatient programs for total hip and knee arthroplasty (THA/TKA) is also attractive due to increased capacity and bed availability, as well as reduced length of stay ${ }^{4}$ and health care costs 5,6 . However, the main objective for transitioning to an ambulatory practice should be to improve the recovery process until it becomes safe for the patients to return home on the day of surgery. ${ }^{7}$ To achieve this objective, outpatient programs should be developed based on enhanced recovery after surgery (ERAS) principles that focus on optimizing all aspects of perioperative care to ensure effective, secure and rapid recovery. ${ }^{8,9}$ When compared to standard (STD)-inpatient care, some ERASoutpatient TJA programs have shown to enhance both patient safety and effectiveness of care: two of the three pillars of quality of care as described by the National Health Service. ${ }^{10,11,12,13}$ However, little evidence is available with respect to the third pillar: patient experience. Yet, optimizing the clinical experience is as important for providing high quality care as ensuring patient safety and delivering effective care. ${ }^{14}$ Furthermore, excellence in all three pillars must be achieved in order to offer high-quality care. ${ }^{13,14}$ We conducted a study in 48 individuals with staged bilateral THA or TKA who experienced both STD-inpatient and ERAS-outpatient programs. We found that the ERAS-outpatient program reduced complication rates by more than $50 \%(2.1 \mathrm{vs} 4.4, \mathrm{p}<0.001)$ and minimized early post-operative opioid consumption ( $9.3 \mathrm{vs} 26.5 \mathrm{mg}$ of morphine equivalent, $\mathrm{p}<0.001$ ). In addition, patients walked, climbed stairs, showered, performed activities of daily living, practised sports, and went back to work sooner after the ERAS-outpatient joint replacement $(p<0.001)$. Moreover, patients were more satisfied with hospital stay, pain management, recovery, wound care and had overall a better care experience with the ERAS-outpatient program ( $<$ 0.002). ${ }^{12}$ The within-subject design used provided a direct comparison of both programs and limited most interpersonal characteristics bias that might act as potential confounders, thus enhancing the comparison's validity regarding the patient experience. ${ }^{15,16}$ Nevertheless, we acknowledge that the patient experience is a complex concept representing "the sum of all interactions, shaped by an organization's culture, that influence patient perceptions, across the continuum of care" and that understanding the patient experience or factors enhancing it, goes beyond quantitative assessment alone. ${ }^{17,18}$ Therefore, using the same bilateral patients cohort, this study's overarching objective was to gain a more in-depth understanding of the patient experience for both STDinpatient and ERAS-outpatient total joint arthroplasty by combining the strengths of quantitative and qualitative approaches. ${ }^{19}$ Specifically, we aimed to explore the patient experience of ERAS-outpatient programs compared to STD-inpatient programs and to identify elements that could optimize patients' experience. We also sought to determine whether and how patient characteristics, clinical outcomes and satisfaction of care components are associated with patients' experience. 


\section{Methods}

\section{Study Design, Patient Selection and Characteristics}

This study used a convergent mixed methods single subject design (Quan + Qual) to gain a more in-depth understanding of patient experience for both STDinpatient and ERAS-outpatient total joint arthroplasty. In October 2020, in conjunction with another study, ${ }^{12}$ we recruited the first 50 adult patients who underwent both programs for the same primary surgery on contralateral articulations (THA-THA or TKA-TKA) in a Canadian tertiary hospital and who were able to communicate in French or English. Out of all eligible participants, 48 gave an informed written consent and enrolled in the study; two patients declined because they were not interested in participating. The study was conducted in accordance with the Declaration of Helsinki and this study was approved by our Institutional Review Board (Comité d'éthique de la recherche du CIUSSS de l'Est-de-l'Ile-de-Montréal \#2021-2420). Full details of participants' characteristics were presented in a previous paper ${ }^{12}$ and are summarized in Table 1.

Table 1

Patients' characteristics

\begin{tabular}{|llll|}
\hline & ERAS-outpatient & STD-Inpatient & P-value \\
\hline Number of participants & 48 & & \\
\hline $\begin{array}{l}\text { Gender } \\
\text { (Men: Women) }\end{array}$ & $27(56.2 \%): 21(43.8 \%)$ & & \\
\hline Average Age at Surgery & $60.0(45.0-76.0,7.8)$ & $52.9(33.0-70.0,8.3)$ & $<0.001^{\text {a }}$ \\
\hline Average Body Mass Index & $28.1(20.7-37.6,4.1)$ & $28.3(19.3-35.0,4.0)$ & $0.638^{\text {a }}$ \\
\hline ASA Physical Status & $17(35.4 \%)$ & $25(52.1 \%)$ & $0.007^{\text {b }}$ \\
\hline 1 & $30(62.5 \%)$ & $23(47.9 \%)$ & \\
\hline 2 & $1(2.1 \%)$ & 0 & \\
\hline 3 & & & \\
\hline Intervention & $36(75.0 \%)$ & & \\
THA & $12(25.0 \%)$ & & \\
TKA & & & \\
\hline a: Paired T-test & & & \\
\hline
\end{tabular}

b: Marginal homogeneity test

\section{STD-Inpatient and ERAS-Outpatient Programs Description}

In both programs, patients met with several health professionals (orthopaedic surgeon, anesthesiologist, internist, nurse, and physiotherapist) weeks before the day of surgery. In addition to the standard preparation care, an informative group session regarding specificity of the outpatient recovery process was offered in the ERAS-outpatient program. Recommendations before surgery were the same for both programs except that patients had to fast at midnight before the STD-inpatient procedure, whereas they could drink clear sugary liquids up to 2 hours before the ERAS-outpatient operation. Perioperative care provided in the STD-inpatient program varied according to the patient's characteristics and surgeon's preferences, whereas it was standardized in the ERASoutpatient program so every patient received the same interventions. Surgical approach and types of implants used were identical between programs. The first physiotherapy session aimed to be performed on postoperative day 1 in the STD-inpatient program and in the first 4-6 hours after surgery in the ERASoutpatient program. In the STD-inpatient program, patients were expected to be hospitalized 1-3 days for a THA and 3-5 days for a TKA whereas they were expected to be discharged the same day of surgery or in $<24$ hours, respectively, with the ERAS-outpatient program. Regardless of the program, all patients returned home after discharge, followed the same rehabilitation program, and received similar home care services. STD-inpatient surgeries took place between 2000 and 2018 and were all performed before the ERAS-outpatient surgeries, which were performed between 2017 and 2020 . Detailed description of both ERAS-outpatient and STD-inpatient programs were previously presented by Vendittoli et al. ${ }^{10}$ and Hardy et al. ${ }^{12}$

\section{Data Collection}

Patient characteristics (gender, age, body mass index, medical comorbidities according to the American Society of Anesthesiologist Physical Status classification and type of intervention [THA or TKA]) and postoperative data (pain on a numeric rating scale (NRS) [0-10], opioid consumption in milligram morphine equivalent, numbers of complications measured with the Clavien-Dindo classification, complications' morbidity rated with the Comprehensive Complication Index, numbers of unplanned episodes of care, length of stay in hours and time in days to reach functional recovery landmarks at the hospital) were collected retrospectively from medical records. The time to reach functional recovery landmarks at home in days or weeks, both patient overall care experience and patient satisfaction of care components (preparation before surgery, hospital stay, homecare, pain management, postoperative recovery, wound care, overall result) on visual analog scale (VAS) [0-100] and patient recommendation for a specific TJA program were collected prospectively at the last follow-up (9 months to several years after surgeries) through the Patient Experience questionnaire which was developed to enable both quantitative and qualitative assessment of patients' TJA experience [Supplementary Material-Appendix A]. Indeed, when patient overall care experience and patient 
satisfaction of care components on VAS differed between programs, patients were asked open-ended questions to provide a deeper understanding of their experience: "If your satisfaction of ... differs between surgeries, please explain why." In addition, participants were invited to explain their answers when questioned about which program they would recommend: "What surgical protocol (way of doing things) between the one you had on the left side and the one you had on the right would you recommend for a hip/knee replacement? Please explain why." Moreover, they were invited to suggest any improvement they thought that could be made to the programs to enhance their experience: "How could we improve patients' experience for hip/knee replacement? Please give your suggestions." To prevent any potential wording bias related to the names of the programs "Enhanced Recovery after Surgery" versus "Standard", all questions referred to "Right" and "Left" sides instead. Once the survey completed, answers were categorized to the appropriate program (ERAS-outpatient or STD-inpatient) before proceeding to analyses. All the data collected were de-identified and stored in a secured REDCap database [Research Electronic Data Capture, Vanderbilt University, TN, USA]

\section{Data Processing and Analysis}

\section{Qualitative Data}

All text data gathered through open-ended questions in the Patient Experience questionnaire were qualitatively analyzed in QDA Miner [QDA Miner, Version 5.0, Provalis Research, Montreal, QC, CAN] using a reflexive thematic analysis inspired by Braun \& Clark. ${ }^{20}$ First, two authors (A. Hardy and J.G.H.) read the entire data set many times to become familiar with it. They recorded their thoughts in a research audit trail. Second, based on an inductive and semantic approach, both investigators generated initial codes independently for five participants. Then, they compared their initial codes, made modifications if needed, and elaborated a common codebook based on their consensus. Third, they repeated this process for five more participants to assure trustworthiness regarding the analytical approach. Fourth, A. Hardy coded the data of remaining participants, and afterwards grouped codes into broader categories and developed overarching themes. Fifth, he created a thematic map to visualize the relationships between themes and to sort main themes from secondary ones. Sixth, the representation of results was debriefed and discussed with all investigators which led A. Hardy to reread the entire data set and refined themes to form a coherent whole that truly captured the essence of the participants' experiences. When refinements stopped producing significant improvements, final themes were approved by the whole research team.

\section{Quantitative Data}

Relations between patient overall care experience on the VAS [0-100] and continuous data on patient characteristics, clinical outcomes and patient satisfaction of care components were analyzed with Pearson or Spearman bivariate correlations. Point-biserial correlations were realized when patient characteristics and clinical outcomes data were dichotomous. A two-sided alpha level of significance of 0.05 was used and all tests were performed with SPSS - 25 [IBM SPSS Statistics for Macintosh, Version 25.0. IBM Corp Armonk, NY, USA].

\section{Quantitative and Qualitative Data Integration}

The qualitative and quantitative data were first analyzed separately. Then, they were integrated together in the discussion and compared to the literature to provide a more in-depth understanding of patient experience for both STD-inpatient and ERAS-outpatient total joint arthroplasty. ${ }^{21}$

\section{Results \\ Qualitative Results}

The qualitative analysis allowed to identify themes that provided a better understanding of the patient experience during TJA. One theme was commonly present among both programs and across the entire care episode: Support makes the difference-for better and for worse. Three other themes captured improvements in the patient experience due to the ERAS-outpatient program compared to STD-inpatient one: 1) Minimizing inconvenience, 2) Home sweet home and 3) Returning to normal function and activities. Finally, another theme captured improvements that could be made to the patient experience: Room for Improvement. Quotes from Francophone participants were translated into English by a native English speaker.

\section{Support Makes the Difference for Better and for Worse}

No matter the phase of care and the program, participants spontaneously evoked their appreciation for benefiting from a well-framed structure from beginning to end. They felt better supported and confident when healthcare providers identified themselves and answered their questions. They appreciated healthcare providers who are good listeners and valued receiving clear information, so they were not left in the unknown.

"For me, everything was perfect, the information was clear, the care from start to finish, everything was well managed, I never felt in the unknown." (Patient 40)

On the other hand, they were dissatisfied when the first contact with the healthcare team was judged as non-professional or when their surgeon did not visit them after the intervention.

Participants also discussed how the support provided on the ward after surgery affected their experience. Some disliked their STD-inpatient experience because they thought that postoperative care was disorganized and of poor quality. They observed that the staff seemed overwhelmed and felt that they did not care about them as several patients overheard the staff arguing about who will assume responsibility for their care. Others shared how they felt let down because they experienced complications and healthcare providers took a long time to acknowledge and address them. A few patients even reported that family members would have done better. In contrast, participants appreciated the competency and professionalism of healthcare workers dedicated to the outpatient ward and some even expressed they felt pampered. Participants also discussed the support they received at home after surgeries. Most of them enjoyed home care services provided by nurses and physiotherapists in both programs. They said they appreciated the professionalism of home care 
professionals as they took all the time needed to provide care, answer questions and to refer to other healthcare professionals when required. Some participants even said that having a dedicated member of the orthopaedic team made available to answer their questions and tamper their fears and anxieties, was a huge plus. On the other hand, a few participants had mixed feelings about the quality of care they received. These participants considered that the healthcare workers who came to assist them were incompetent and unprofessional. They stated that home care providers changed personnel often, were not familiar with the patient's file, did not follow the instructions given by the surgical team, did not detect complications, were unable to answer their inquiries and sometimes did not show up to provide the prescribed care.

"The unprofessionalism of the CLSC ${ }^{1}$, meaning they did not take notice of my file, were unable to answer questions, changed personnel each time, did not follow the post-operation directives. For example: they removed the special bandage (bee hole) without respecting the time frame and in addition to the bandage being only stained the equivalent of 2 bee holes." (Patient 39)

A few participants even felt abandoned after their STD-inpatient TJA because they perceived that the home care services provided were less extensive compared to those they received in the ERAS-outpatient program. Overall, patients expressed the importance of support from the health care team throughout the entire care process in both programs. However, when participants reported differences between programs, they usually felt better supported in the ERASoutpatient program because they benefitted from workers dedicated to this program in the ward.

"Left hip, day surgery protocol. AWESOME! Qualified and dedicated staff for day surgeries. Always someone close by to make sure everything goes well. I had questions about my medication, the orthopaedic nurse referred me to a pharmacist right away and I quickly got the right advice. I felt like I was being treated like a princess because the people were so attentive." (Patient 34)

\section{Minimizing Inconvenience}

Following both interventions, some patients experienced minor complications. However, they noted differences between programs regarding prevention and management of adverse events.

For pain management, participants preferred the ERAS-outpatient program because being home sooner allowed them to take analgesics as prescribed without having to wait for the hospital staff, unlike in the STD-inpatient program. In addition, their pain was better relieved with less medication with the ERASoutpatient program. Patients also liked the opioid-sparing analgesic modalities of the ERAS-outpatient program because it prevented side effects associated with narcotics, including vomiting or constipation, and alleviated their fear of developing an addiction. Moreover, patients noted that staples used to close the surgical wound were replaced by sutures and tissue adhesive in the ERAS-outpatient program which eliminated the inconvenience and pain of having them removed. Moreover, participants reported that staples caused bothersome side effects, such as wound discharge and itching, which were not reported with the combination of sutures and tissue adhesive. Many participants preferred the use of tissue adhesive in the ERAS-outpatient program because it not only enabled them to shower sooner, it also allowed their wounds to heal better and more quickly, resulting in scars that were smaller and more aesthetically appealing.

"With the glue, it's fantastic, no discharge, you can shower faster, the scar heals faster and looks better" (Patient 40).

Patients often reported leg edema after surgery, although they described it as less bothersome after the ERAS-outpatient procedure. Some participants noted that they did not experience urinary retention after their ERAS-outpatient surgery, unlike the STD-inpatient one. Overall, patients reported the type of anesthesia as being the main difference between the two programs. Some stated that, unlike after the STD-inpatient surgery, they did not suffer from prolonged motor blockade after ERAS-outpatient surgery. Others reported that the epidural-sedation combination used in ERAS-outpatient surgery was as efficient as general anesthesia used in STD-inpatient surgery, but better tolerated with fewer side effects. They added that only being sedated enabled waking up and recovering more quickly after the surgery.

"Easier to wake up from surgery (ERAS-outpatient) and go home the next day, less sick. I was much better in general when I came out of the operating room than at the first operation (STD-inpatient)." (Patient 10)

Globally, participants expressed that postoperative inconveniences were better prevented or managed in the ERAS-outpatient program.

\section{Home Sweet Home}

Most patients appreciated the reduced length of stay in hospital and the possibility of going home quickly with the ERAS-outpatient program. Participants were thrilled to be discharged home rapidly after surgery because they felt the ward can be a harsh setting in which to recover. They evoked the difficulty of getting a good night of sleep because of the noise, their lack of interest in the food served and the anxiety-inducing atmosphere of the environment. For them, leaving the hospital as early as possible was truly appreciated.

"The less time spent in hospital the better! Long live day surgery!" (Patient 45)

However, for other patients, the expectation to go home only hours after surgery was at first a source of anxiety. Nevertheless, they were reassured about going home early because a caregiver would be with them. Participants enjoyed rapidly regaining the comfort of their home where they could enjoy a dinner with their relatives on the same day of their surgery and benefit from the tranquility of their own environment to fully rest and recover. Even when they reported excellent care at the hospital for their first surgery, patients found convalescence was easier and better for the mind when carried out at home.

"Despite the good care of the nursing staff, recovery is easier at home, in my environment, much easier and better for the spirit." (Patient 25)

\section{Returning to Normal Function and Activities}


Most participants reported major differences between programs in the time and effort required to return to normal function. They expressed their delight in performing activities of daily living, such as walking and climbing stairs on the day of surgery with the ERAS-outpatient program, which was not the case with the STD-inpatient program. They enjoyed being able to walk without aid rapidly after the ERAS-outpatient surgery. Some even shared their astonishment in being able to go shopping and going back to work only a couple of days later.

"For the left leg with the day surgery, the recovery was really super-fast. 3 days later I was at the grocery shop and the next day I was going to the office! Much faster than the first operation which still went very well in terms of recovery." (Patient 45)

Participants noted that the overall recovery process was generally quicker and easier following the ERAS-outpatient TJA because of what they perceived as enhanced supervision and better adapted exercises. They noted that fewer physiotherapy sessions were needed to recover compared to the STD-inpatient intervention.

"For the right side (STD-inpatient) much more time in physio 1-2 months, whereas the left side, (ERAS-outpatient) 3 visits in physio and finished thereafter." (Patient 3)

Some patients did not even feel the need to consult with outpatient physiotherapy following the care provided at home in the first days. However, in both programs, some patients experienced obstacles such as pain and deficits in strength and flexibility that hampered their functional recovery. For very few patients, these difficulties persisted and negatively tainted their experience, whereas most patients were happy with the overall results of both TJA.

\section{Room for Improvement}

Many patients wrote that their TJA experiences were perfect, especially for the ERAS-outpatient program. Nevertheless, improvements to TJA programs were highlighted by some patients. Patients hoped solutions could be found to reduce waiting time before surgery. For the preparation phase, patients suggested to present patients' testimonies of previous experiences and to further explain the patient's role in the process to optimize recovery. They further recommended that surgeons come visit their patients in the recovery room, as some did not see their surgeon after the surgery. Moreover, patients suggested that having more physiotherapy sessions, as needed, would be beneficial.

"Having more physiotherapy at home if needed: I believe this is the key after a successful surgery" (Patient 8)

Also, participants recommended that communication between the orthopaedic team and home care teams be improved to convey more coherent instructions.

"Improved communication between the hospital physio and the CLSC ${ }^{2}$ physio. The hospital physio explained to me that with the new protocol (ERASoutpatient) I could start walking, climbing stairs and even riding a stationary bike faster whereas the CLSC asked me to wait." (Patient 1)

Finally, they advocated to maintain having a dedicated resource person from the orthopaedic team easily reachable by phone to answer inquiries.

\section{Quantitative Results}

Bivariate Correlations of Patient Overall Care Experience with Patient Characteristics, Clinical Outcomes and Patient Satisfaction of Care Components

The bivariate correlation between patients' overall care experience and patient characteristics and clinical outcomes showed only poor association $r_{\mathrm{s}}= \pm$ [.000-0.299] that was not statistically significant ( $p>0.05$; Table 2). The positive associations between patients' overall care experience and satisfaction of care components in the ERAS-outpatient group were found to be fair $r_{s}=+[0.400-0.599]$ to moderate $r_{s}+[0.600-0.799]$ and were all considered statistically significant $(p<0.01$; Table 3$)$. The spearman correlation coefficients between these same variables in the STD-inpatient group showed generally weaker positive associations, ranging from poor and non-statistically significant $(p \geq 0.108)$ for preparation and wound care to fair and statistically significant for hospital stay, home care, pain management, postoperative recovery and overall results $(p<0.014)$. 
Table 2

Relations between patient overall care experience and patients' characteristics and clinical outcomes

\begin{tabular}{|c|c|c|c|c|}
\hline & ERAS-Outpatient & & STD-Inpatie & \\
\hline Patient characteristics & $\begin{array}{l}\text { Spearman } \\
\text { correlation } \\
\text { coefficient }\end{array}$ & $\begin{array}{l}\text { P- } \\
\text { value }\end{array}$ & $\begin{array}{l}\text { Spearman } \\
\text { correlation } \\
\text { coefficient }\end{array}$ & $\begin{array}{l}\mathrm{P}- \\
\text { value }\end{array}$ \\
\hline Gender & 0.044 & 0.771 & 0.137 & 0.359 \\
\hline Age at surgery & 0.148 & 0.321 & 0.158 & 0.288 \\
\hline Body Mass Index & 0.152 & 0.309 & 0.136 & 0.368 \\
\hline ASA Physical Status & 0.163 & 0.273 & 0.089 & 0.553 \\
\hline Intervention (THA/TKA) & 0.091 & 0.545 & -0.188 & 0.205 \\
\hline \multicolumn{5}{|l|}{ Postoperative variables } \\
\hline $\begin{array}{l}\text { Pain in the recovery room } \\
\text { (NRS 0-10) }\end{array}$ & 0.002 & 0.99 & -0.071 & 0.651 \\
\hline $\begin{array}{l}\text { Pain in the ward, surgery day } \\
\text { (NRS 0-10) }\end{array}$ & 0.235 & 0.112 & -0.043 & 0.779 \\
\hline $\begin{array}{l}\text { Mean Opioid Consumption in Morphine Milligram Equivalents in the First } 8 \text { Hours After } \\
\text { Surgery }\end{array}$ & -0.163 & 0.275 & -0.087 & 0.56 \\
\hline Complications: All Grades & 0.060 & 0.689 & 0.020 & 0.892 \\
\hline Complications: Grade 1 & 0.053 & 0.721 & 0.085 & 0.572 \\
\hline Complications: Grade 2 & -0.037 & 0.806 & -0.273 & 0.063 \\
\hline Comprehensive Complication Index & -0.023 & 0.876 & -0.163 & 0.273 \\
\hline Emergency Care Unit visits without intervention & -0.239 & 0.106 & -0.039 & 0.794 \\
\hline Emergency Care Unit visits with interventions & -0.015 & 0.922 & 0.022 & 0.882 \\
\hline Clinic consultations without intervention & -0.057 & 0.706 & 0.034 & 0.822 \\
\hline Clinic consultations with interventions & & & -0.257 & 0.082 \\
\hline First rise/standing (in days) & 0.232 & 0.117 & 0.192 & 0.218 \\
\hline Walking (in days) & 0.058 & 0.71 & 0.144 & 0.382 \\
\hline $\begin{array}{l}\text { Going up and down the stairs } \\
\text { (in days) }\end{array}$ & 0.205 & 0.181 & -0.003 & 0.985 \\
\hline Length of stay (in hours) & 0.095 & 0.527 & -0.162 & 0.281 \\
\hline First shower (in days) & -0.103 & 0.497 & -0.245 & 0.104 \\
\hline $\begin{array}{l}\text { Walking without technical aid } \\
\text { (in days) }\end{array}$ & -0.005 & 0.972 & -0.106 & 0.484 \\
\hline Going up and down the stairs without technical aid (in days) & -0.014 & 0.923 & -0.031 & 0.836 \\
\hline ADLs: dressing, toileting, walking indoor alone, etc. (in days) & -0.19 & 0.201 & -0.174 & 0.248 \\
\hline iADLs: cooking, cleaning, shopping, etc. (in days) & -0.007 & 0.965 & -0.109 & 0.472 \\
\hline Mild physical activities: cycling, swimming, walking, etc. (in weeks) & -0.085 & 0.584 & 0.088 & 0.567 \\
\hline $\begin{array}{l}\text { Intense physical activities: running, playing tennis, skiing, etc. } \\
\text { (in weeks) }\end{array}$ & 0.28 & 0.166 & 0.106 & 0.59 \\
\hline $\begin{array}{l}\text { Return to light-duty work } \\
\text { (in weeks) }\end{array}$ & 0.285 & 0.121 & 0.097 & 0.562 \\
\hline Return to regular work without limitation (in weeks) & 0.193 & 0.344 & 0.069 & 0.707 \\
\hline
\end{tabular}


Table 3

Satisfaction of care components and relations with patient overall care experience

\begin{tabular}{|c|c|c|c|c|c|c|c|}
\hline \multirow[b]{2}{*}{ Variables } & \multicolumn{3}{|c|}{ Satisfaction of care components ${ }^{12}$} & \multicolumn{2}{|l|}{ ERAS-outpatient } & \multicolumn{2}{|l|}{ STD-inpatient } \\
\hline & ERAS-outpatient & STD-inpatient & $\begin{array}{l}\mathrm{P} \text { - } \\
\text { value }\end{array}$ & $\begin{array}{l}\text { Spearman correlation } \\
\text { coefficient }\end{array}$ & $\begin{array}{l}\mathrm{P} \text { - } \\
\text { value }\end{array}$ & $\begin{array}{l}\text { Spearman correlation } \\
\text { coefficient }\end{array}$ & $\begin{array}{l}\mathrm{P} \text { - } \\
\text { value }\end{array}$ \\
\hline Preparation & $\begin{array}{l}94.4(60.0-100.0 \\
9.8)\end{array}$ & $\begin{array}{l}92.9(53.0- \\
100.0,11.6)\end{array}$ & 0.356 & .453 & 0.001 & 0.238 & 0.108 \\
\hline Hospital stay & $\begin{array}{l}96.0(50.0-100.0 \\
9.0)\end{array}$ & $\begin{array}{l}84.6(0.0-100.0, \\
23.1)\end{array}$ & $\dot{0} 001$ & .614 & $\dot{0} 001$ & .450 & 0.001 \\
\hline Homecare & $\begin{array}{l}89.0(11.0-100.0 \\
20.2)\end{array}$ & $\begin{array}{l}91.8(39.0-100.0, \\
13.2)\end{array}$ & 0.678 & .661 & $\dot{0} 001$ & .411 & 0.004 \\
\hline $\begin{array}{l}\text { Pain } \\
\text { management }\end{array}$ & $\begin{array}{l}93.2(18.0-100.0 \\
13.5)\end{array}$ & $\begin{array}{l}86.4(0.0-100.0 \\
21.4)\end{array}$ & 0.002 & .582 & $<.001$ & .357 & 0.014 \\
\hline $\begin{array}{l}\text { Functional } \\
\text { recovery }\end{array}$ & $\begin{array}{l}95.5(70.0-100.0 \\
6.8)\end{array}$ & $\begin{array}{l}84.4(1.0-100.0 \\
19.9)\end{array}$ & $\dot{0} 001$ & .486 & 0.001 & .374 & 0.01 \\
\hline $\begin{array}{l}\text { Wound } \\
\text { management }\end{array}$ & $\begin{array}{l}94.8(18.0-100.0 \\
13.2)\end{array}$ & $\begin{array}{l}85.1(0.0-100.0 \\
18.2)\end{array}$ & $<0.001$ & .614 & $<0.001$ & 0.124 & 0.405 \\
\hline Overall Result & $\begin{array}{l}95.9(70.0-100.0 \\
7.6)\end{array}$ & $\begin{array}{l}91.8(0.0-100.0 \\
17.3)\end{array}$ & 0.187 & .578 & $\begin{array}{l}< \\
0.001\end{array}$ & .420 & 0.003 \\
\hline
\end{tabular}

\section{Footnote:}

[1] Centres locaux de services communautaires (CLSC) are the homecare providers.

[2] Centres locaux de services communautaires (CLSC) are the homecare providers.

\section{Discussion}

This study specifically aimed to explore the patient experience of ERAS-outpatient programs compared to STD-inpatient programs and to identify elements that could optimize patients' experience. It also sought to determine whether and how patient characteristics, clinical outcomes and satisfaction of care components are associated with patients' experience. The qualitative analysis demonstrated that the quality of support received by the patients throughout the episode of care is important to their TJA experience, and that overall, patients had a better experience with the ERAS-outpatient program because they felt better supported by staff dedicated to ambulatory surgery in the ward, they experienced less postoperative inconvenience, went home sooner and recovered more quickly. Furthermore, patients suggested that their TJA experience could possibly be enhanced by improving the information given in the preparation phase, providing more postoperative physiotherapy sessions at home if needed and ensuring better coherence of care between orthopaedic and homecare teams. The bivariate analyzes revealed no statistically significant relationship between patient experience and patient characteristics or clinical outcomes and weak to moderate positive correlations between patient experience and satisfaction with pain management, hospital stay, postoperative recovery, homecare and overall results. Taken separately, these findings enhance our comprehension of the patient TJA experience. Nevertheless, this study's overarching objective was to gain a more in-depth understanding of the patient experience for both STD-inpatient and ERAS-outpatient total joint arthroplasty by combining the strengths of quantitative and qualitative approaches. ${ }^{19}$ Consequently, qualitative and quantitative results were integrated together and compared to the literature in the following paragraphs.

\section{Patients Characteristics}

Some studies on TJA found that age and certain comorbidities are predictors of postoperative satisfaction which could have altered patient experience. ${ }^{22,23,24,25}$ Nevertheless, the impact of these patient characteristics is still controversial as many other studies on TJA, on the other hand, found no significant correlations. ${ }^{15,26,27,28}$ Our findings corroborated the latter as the bivariate analyses did not find any significant correlation between patient experience and patient characteristics. Moreover, in the qualitative phase, participants did not mention how their age or their comorbidities affected their experience throughout the episode of care. When taken together, these findings suggest that patients' characteristics are not associated with patient experience and that the enhanced patient experience in the ERAS-outpatient programs was unlikely to be associated with the difference in patients' age and comorbidities between surgeries.

\section{Clinical Outcomes}

In this cohort of patients, it was shown that compared to the STD-inpatient care, the ERAS-outpatient program resulted in fewer complications, similar postoperative pain with less opioid consumption and sooner functional recovery. ${ }^{12}$ These findings were corroborated by the present qualitative analysis and are also in line with the conclusion of many studies that showed that prevention of complications, ${ }^{23,28,29}$ well-relieved pain ${ }^{27,28,29}$ and rapid recovery ${ }^{28,29,30,31}$ after TJA are predictors of better patient satisfaction. However, we did not find any significant correlation between these clinical outcomes and the patient experience nor did other studies. ${ }^{32,33}$ These divergent results may be explained by the fact that strengths of relationship with patient experience after TJA are shaped by the focus of the question. ${ }^{25}$ Our question referred specifically to the patients' overall care experience, a complex concept which differs from the overall satisfaction evaluated in the other studies. ${ }^{23,27,28,29,30,31,34}$ Also, the different quantitative and qualitative findings underscored the importance of going 
beyond quantitative assessment alone to truly understand what influences patients' TJA experience. Patients clearly discussed how clinical outcomes, such as adverse events, pain, and functional recovery, affected their experience, although no correlation was found. ${ }^{35}$

\section{Postoperative Recovery}

Sooner and better recovery after surgery is the main goal of ERAS interventions and represented some of the main reasons why patients reported a better TJA experience with the ERAS-outpatient program. ${ }^{36}$ Indeed, satisfaction with postoperative recovery was positively associated with patient overall experience of care. Patients appreciated that the epidural-sedation combination had the advantage over spinal anesthesia alone of eliminating the distress associated with being awake during the operation and that it made them feel better and recover more quickly after surgery. ${ }^{37,38}$ The ERAS-outpatient rehabilitation protocol was even perceived as "enhanced and better adapted", although the only difference with the STD-inpatient program was earlier postoperative evaluation and ambulation. Still, earlier ambulation after TJA was shown to reduce complications and to improve pain, muscles strength, range of motion, gait and overall function in the post-acute phase which may explain why patients enjoyed their recovery more following the ERAS-outpatient surgery. ${ }^{39,40,41,42}$ The rapid mobilization after surgery in the ERAS-outpatient program also contributed to a reduction in the number of rehabilitation sessions needed to reach full functional autonomy which was greatly appreciated by the patients. ${ }^{12,39}$ Thus, by improving the recovery process, the ERAS-outpatient program enhanced the patients' overall TJA experience. Similarly, Johansson Stark et al. ${ }^{43}$ observed that a positive patient experience increased the likelihood of better postoperative recovery. Consequently, as recommended by Doyle et al. ${ }^{14}$, all three pillars of quality of care (patient safety, clinical effectiveness and patient experience) should be viewed as a group and interventions should aim to improve all three to provide high-quality care in TJA programs.

\section{Pain Management}

Pain management is crucial after TJA, as unrelieved pain can negatively affect recovery and patient experience. ${ }^{44,45,46,47,48}$ Indeed, satisfaction with pain management was found to be weakly to fairly positively correlated with patient experience. Rapid and effective pain relief is even more important in outpatient programs as it is a major reason for failure to discharge within the expected time frame. ${ }^{49}$ With outpatient programs, patients become quickly responsible for their pain management and it was shown to often result in unrelieved pain. ${ }^{45,50}$ When self-medicating, patients are often reluctant to take the prescribed analgesics because they are not sure when to take them or because they are afraid of developing an addiction. ${ }^{45,46,50}$ Despite these additional challenges, our participants preferred the ERAS-outpatient pain management protocol that combined preemptive medication, dexamethasone, epidural-sedation anesthesia, local infiltration analgesia and multimodal analgesics over the STD-inpatient modalities that often included narcotics, because they felt it was more effective and they had less concern about developing a dependency. The patients also enjoyed being able to quickly self-manage their analgesics consumption in the post-acute phase with the ERAS-outpatient program because it enabled them to alleviate their pain rapidly, without having to wait for nursing staff. This was shown to be strongly associated with better overall pain relief and lower need for additional analgesia. ${ }^{51}$ Compared to other studies' findings, ${ }^{45,46,50}$ the superior coping abilities with self-pain management in our study could be due to the extensive education provided to patients in the preparation phase and before discharge, as it enabled them to be knowledgeable and confident with the postoperative process. ${ }^{52}$ Furthermore, as has been suggested by the patients, the overall care experience would surely benefit from former patients sharing their advice and providing additional information. This would empower patients regarding the process of care, thus increasing their confidence in performing perioperative tasks, such as pain management, and improving their recovery. ${ }^{53,54,55}$

\section{Hospital Stay and Homecare}

Satisfaction with hospital stay and homecare were also found to be fairly to moderately positively correlated with patient experience, which is consistent with patients highlighting the quality of care they received at the hospital and at home as important to their experience. Indeed, patients enjoyed their hospital experience in ERAS-outpatient program more mainly because they felt better supported by nurses and physiotherapists who were specialized in outpatient programs. ${ }^{45}$ The support at home was also crucial to the patient experience and most patients expressed a positive homecare experience in both programs because they perceived homecare providers as competent, engaged and interested in helping them recover. Still, a few patients were disappointed by the homecare provided, which they deemed unprofessional or not cohesive with the instructions given by the orthopaedic team, thus leading to uncertainty. ${ }^{45}$ Fortunately, patients benefited from having a caregiver at home in the early postoperative days, which was mandatory to undergo the ERAS-outpatient program, to alleviate the uncertainties of homecare and temper the additional concerns of returning home quickly. ${ }^{56,46}$ Patients also appreciated that, in both programs, a nurse from the orthopedic team was available by phone to answer uncertainties, ease concerns, and provide information which helped to improve the overall provider-patient relationship. ${ }^{57,58}$ The present findings reinforced those of McMurray et al. ${ }^{58}$ stating that relationships between patients and care providers are a key component of the patient experience and that aspects such as caring, empathy, respect and perceived provider expertise all influenced the overall experience. ${ }^{58}$ On the other hand, the poor homecare experience of some patients underscores the importance of improving and standardizing the quality of home care and educating homecare providers on the rehabilitation protocol, in order to provide a consistent message that will improve patient experience and also potentially improve the postoperative outcome. ${ }^{59}$

\section{Wound Care}

Wound care is also a potential significant contributor to the patient experience in both the early and late postoperative phases, as the scar remains a visible reminder of their journey. ${ }^{60}$ In this study, we found that satisfaction with wound care was moderately positively associated with patient experience for the ERAS-outpatient surgery but not for the STD-inpatient program. This finding is probably secondary to the distinct interactions of each program that generated a different perception by the patients of the importance of wound care in their care experience. 
Still, patients expressed how the combination of subcuticular sutures and tissue adhesive of the ERAS-outpatient program allowed them to shower sooner, avoid the inconvenience of staples (removal and wound discharge) and have better cosmetic results, enhanced their experience.61,62,63 Therefore, optimizing wound care should be considered important to the patients' TJA experience, especially in outpatient program, where minimizing postoperative inconvenience is crucial to prevent additional concerns and additional episodes of care, which could negate some benefits of outpatient procedures.

\section{Overall Results}

Black et al. ${ }^{64}$ observed a weak positive correlation between patient-reported experience measures (PREMs) and patient-reported outcome measures (PROMs) at 6 months after a TJA. Similarly, we found that satisfaction with overall results at the last follow-up was fairly to moderately positively correlated with patient experience. However, patients did not express a difference between their ERAS-outpatient and STD-inpatient surgery overall results, which is in line with the similar PROMs at the last follow-up. ${ }^{12}$ Thus, although there is a positive correlation between patient experience and satisfaction with TJA overall results, it is unlikely that it contributed much to the better overall patient experience found with the ERAS-outpatient program.

\section{Study Limitations}

The present results must be interpreted taking into consideration this study's limitations. First, while bivariate analyses quantify the strengths of the relationship between two variables, they do not provide information regarding causality or direction. Nevertheless, this limitation was mostly overcome by the mixed methods design which enable to obtain a complete understanding of the patient experience and its associated factors by integrating qualitative data to the quantitative data. Second, unlike the case with interviews, our survey-based instrument did not allow us to ask sub-questions that may have allowed to further deepen the participants' points of view. However, the large sample size by qualitative research standards was more than sufficient to gather a great variety of perspectives and experiences. Third, all surgeries were performed in the same Canadian tertiary centre and the results may not be generalizable to other settings. Fourth, all STD-inpatient surgeries were performed before the ERAS-outpatient ones. Nevertheless, the comparison of patient satisfaction between the first and the second procedure in bilateral asynchronous TKA showed no difference. ${ }^{65}$ Fifth, participants had to recall their surgeries to answer the Patient Experience questionnaire. Yet, many strategies were put in place to minimize the impact of patient recall: participants were their own comparison, they were blind to the study hypothesis, they could take as much time as they needed to answer, data collection was standardized and done simultaneously for both programs. ${ }^{66}$

\section{Conclusion}

This convergent mixed methods study demonstrated that support throughout the care episode is key to the patient experience regardless of the program. Furthermore, this study addressed a lack in the literature by being the first study, to our knowledge, to demonstrate that ERAS-outpatient arthroplasty programs offered an enhanced patient experience compared to standard-inpatient practice by reducing postoperative inconvenience, offering enhanced pain management and dedicated support in the ward, enabling to go home sooner and to recover and regain function sooner. Patients' experience of TJA could be further enhanced by optimizing the information provided, the rehabilitation program and the coherence between care teams.

\section{List Of Abbreviations}

ERAS: Enhanced Recovery after Surgery

PREMs: Patient reported experience measures

PROMs: Patient Reported Outcome Measurement Study

NRS: Numeric rating scale

THA: Total hip arthroplasty

TJA: Total joint arthroplasty

TKA: Total knee arthroplasty

STD: Standard

\section{Declarations}

\section{Ethics approval and consent to participate}

The ethical approval for this study was obtained from the Comité d'éthique de la recherche du CIUSSS de l'Est-de-l'île-de-Montréal \#2021-2420. The informed written consent was obtained for all participants approved by Comité d'éthique de la recherche du CIUSSS de l'Est-de-I'Île-de-Montréal. The study was conducted in accordance with the Declaration of Helsinki.

\section{Consent for publication}

Not applicable

\section{Availability of data and materials}


The datasets used and/or analysed during the current study are available from the corresponding author on reasonable request.

\section{Competing interests}

P-A. V. reports royalties from Microport, grants, personal fees and others from Stryker, grants, personal fees and others from Johnson and Johnson, and grants from Zimmer; all outside the submitted work. All authors declare that they have no competing interests.

\section{Funding}

This research received unrestricted funding from the Maisonneuve-Rosemont Hospital Foundation.

\section{Authors' contributions}

A. Hardy as the first author was involved in conceptualization, data curation, formal analysis, investigation, methodology, project administration, resources and software management, validation, visualization and writing the original draft. J.H.G. contributed to conceptualization, data curation, formal analysis, investigation and validation. A.H., K.P. \& F.D. helped with conceptualization, methodology, supervision, and validation. P-A.V. as senior author contributed to conceptualization, formal analysis, investigation, methodology, project administration, resources, supervision, validation and writing the original draft. All authors contributed to final editing and review of the manuscript. All authors have read and approved the final version submitted.

\section{Acknowledgements}

We thank Serge Marquis and Margaux Courgeon for helping with the medical records assessment. A. Hardy thanks the Department of Biomedical Sciences of the Université de Montréal for the excellence scholarship received while conducting this study.

\section{References}

1. Bombardier C, Hawker G, Mosher D. The Impact of Arthritis in Canada: Today and Over the Next 30 Years. www.arthritisalliance.ca

2. Kort NP, Barrena EG, Bédard M, et al. Resuming elective hip and knee arthroplasty after the first phase of the SARS-CoV-2 pandemic: the European Hip Society and European Knee Associates recommendations. Knee Surg Sports Traumatol Arthrosc. 2020;28(9):2730-2746. doi:10.1007/s00167-020-062339

3. Wilson JM, Schwartz AM, Farley KX, Roberson JR, Bradbury TL, Guild GN. Quantifying the Backlog of Total Hip and Knee Arthroplasty Cases: Predicting the Impact of COVID-19. HSS J. Published online November 4, 2020:1-7. doi:10.1007/s11420-020-09806-z

4. Zhu S, Qian W, Jiang C, Ye C, Chen X. Enhanced recovery after surgery for hip and knee arthroplasty: a systematic review and meta-analysis. Postgrad Med J. 2017;93(1106):736-742. doi:10.1136/postgradmedj-2017-134991

5. Büttner M, Mayer AM, Büchler B, Betz U, Drees P, Susanne S. Economic analyses of fast-track total hip and knee arthroplasty: a systematic review. Eur J Orthop Surg Traumatol. 2020;30(1):67-74. doi:10.1007/s00590-019-02540-1

6. Malviya A, Martin K, Harper I, et al. Enhanced recovery program for hip and knee replacement reduces death rate. Acta Orthop. 2011;82(5):577-581. doi:10.3109/17453674.2011.618911

7. Kehlet H, Slim K. The future of fast-track surgery. BJS Br J Surg. 2012;99(8):1025-1026. doi:https://doi.org/10.1002/bjs.8832

8. Vehmeijer SBW, Husted H, Kehlet H. Outpatient total hip and knee arthroplasty. Acta Orthop. 2018;89(2):141-144. doi:10.1080/17453674.2017.1410958

9. Kehlet H. Enhanced Recovery After Surgery (ERAS): good for now, but what about the future? Can J Anesth Can Anesth. 2015;62(2):99-104. doi:10.1007/s12630-014-0261-3

10. Vendittoli P-A, Pellei K, Desmeules F, et al. Enhanced recovery short-stay hip and knee joint replacement program improves patients outcomes while reducing hospital costs. Orthop Traumatol Surg Res. 2019;105(7):1237-1243. doi:10.1016/j.otsr.2019.08.013

11. Goyal N, Chen AF, Padgett SE, et al. Otto Aufranc Award: A Multicenter, Randomized Study of Outpatient versus Inpatient Total Hip Arthroplasty. Clin Orthop. 2017;475(2):364-372. doi:10.1007/s11999-016-4915-z

12. Hardy A, Courgeon M, Pellei K, Desmeules F, Loubert C, Vendittoli P-A. Improved Clinical Outcomes of Outpatient Enhanced Recovery Hip and Knee Replacements in Comparison to Standard Inpatient Procedures: A Study of Patients Who Experienced Both. Submitt BMC Musculoskelet Disord J Submiss ID F3f6a9c2-256c-4318-85e9-629752dda2. Published online April 13, 2021. doi:10.21203/rs.3.rs-418395/v1

13. Darzi, Lord. High Quality Care For All.; 2008. https://webarchive.nationalarchives.gov.uk/20130105053023/http://www.dh.gov.uk/en/Publicationsandstatistics/Publications/PublicationsPolicyAndG

14. Doyle C, Lennox L, Bell D. A systematic review of evidence on the links between patient experience and clinical safety and effectiveness. $B M J$ Open. 2013;3(1):e001570. doi:10.1136/bmjopen-2012-001570

15. Batbaatar E, Dorjdagva J, Luvsannyam A, Savino MM, Amenta P. Determinants of patient satisfaction: a systematic review. Perspect Public Health. 2017;137(2):89-101. doi:10.1177/1757913916634136

16. Choi Y-J, Ra HJ. Patient Satisfaction after Total Knee Arthroplasty. Knee Surg Relat Res. 2016;28(1):1-15. doi:10.5792/ksrr.2016.28.1.1

17. Patient Experience 101 - Why? - The Beryl Institute - Improving the Patient Experience. Accessed March 24, 2021.

https://www.theberylinstitute.org/page/PX101_Why

18. Wolf JA. Defining Patient Experience. :15.

19. Creswell JW. Research Design: Qualitative, Quantitative, and Mixed Methods Approaches. 4th ed. SAGE Publications; 2014. 
20. Braun V, Clarke V. Reflecting on reflexive thematic analysis. Qual Res Sport Exerc Health. 2019;11(4):589-597. doi:10.1080/2159676X.2019.1628806

21. Creswell JW, Clark VLP. Designing and Conducting Mixed Methods Research. SAGE Publications; 2017.

22. Farooq H, Deckard ER, Ziemba-Davis M, Madsen A, Meneghini RM. Predictors of Patient Satisfaction Following Primary Total Knee Arthroplasty: Results from a Traditional Statistical Model and a Machine Learning Algorithm. J Arthroplasty. 2020;35(11):3123-3130. doi:10.1016/j.arth.2020.05.077

23. Merle-Vincent F, Couris CM, Schott A-M, et al. Factors predicting patient satisfaction 2 years after total knee arthroplasty for osteoarthritis. Joint Bone Spine. 2011;78(4):383-386. doi:10.1016/j.jbspin.2010.11.013

24. Noble PC, Conditt MA, Cook KF, Mathis KB. The John Insall Award: Patient Expectations Affect Satisfaction with Total Knee Arthroplasty. Clin Orthop Relat Res. 2006;452:35-43. doi:10.1097/01.blo.0000238825.63648.1e

25. Clement ND, Bardgett M, Weir D, Holland J, Gerrand C, Deehan DJ. The rate and predictors of patient satisfaction after total knee arthroplasty are influenced by the focus of the question. Bone Jt J. 2018;100-B(6):740-748. doi:10.1302/0301-620X.100B6.BJJ-2017-1292.R1

26. Clement ND, Walker LC, Bardgett M, et al. Patient age of less than 55 years is not an independent predictor of functional improvement or satisfaction after total knee arthroplasty. Arch Orthop Trauma Surg. 2018;138(12):1755-1763. doi:10.1007/s00402-018-3041-7

27. Hamilton DF, Lane JV, Gaston P, et al. What determines patient satisfaction with surgery? A prospective cohort study of 4709 patients following total joint replacement. BMJ Open. 2013;3(4):e002525. doi:10.1136/bmjopen-2012-002525

28. Bourne RB, Chesworth BM, Davis AM, Mahomed NN, Charron KDJ. Patient Satisfaction after Total Knee Arthroplasty: Who is Satisfied and Who is Not? Clin Orthop Relat Res. 2010;468(1):57-63. doi:10.1007/s11999-009-1119-9

29. Halawi MJ, Jongbloed W, Baron S, Savoy L, Williams VJ, Cote MP. Patient Dissatisfaction After Primary Total Joint Arthroplasty: The Patient Perspective. J Arthroplasty. 2019;34(6):1093-1096. doi:10.1016/j.arth.2019.01.075

30. Baker PN, Rushton S, Jameson SS, Reed M, Gregg P, Deehan DJ. Patient satisfaction with total knee replacement cannot be predicted from pre-operative variables alone. Bone Jt J. 2013;95-B(10):1359-1365. doi:10.1302/0301-620X.95B10.32281

31. Kahlenberg CA, Nwachukwu BU, McLawhorn AS, Cross MB, Cornell CN, Padgett DE. Patient Satisfaction After Total Knee Replacement: A Systematic Review. HSS J. 2018;14(2):192-201. doi:10.1007/s11420-018-9614-8

32. Anhang Price R, Elliott MN, Zaslavsky AM, et al. Examining the Role of Patient Experience Surveys in Measuring Health Care Quality. Med Care Res Rev. 2014;71(5):522-554. doi:10.1177/1077558714541480

33. Bovonratwet P, Shen TS, Islam W, Sculco PK, Padgett DE, Su EP. Is There an Association Between Negative Patient-Experience Comments and Perioperative Outcomes After Primary Total Hip Arthroplasty? J Arthroplasty. Published online January 20, 2021. doi:10.1016/j.arth.2021.01.023

34. Berkowitz B. The Patient Experience and Patient Satisfaction: Measurement of a Complex Dynamic. Online J Issues Nurs. 2016;21(1):1. doi:10.3912/OJIN.Vol21No01Man01

35. LaVela SL, Gallan AS. Evaluation and measurement of patient experience. :11.

36. Kehlet H, Wilmore DW. Evidence-Based Surgical Care and the Evolution of Fast-Track Surgery. Ann Surg. 2008;248(2):189-198. doi:10.1097/SLA.0b013e31817f2c1a

37. Ac K, M E, Al M, S AÖ. "Is that my leg?" patients' experiences of being awake during regional anesthesia and surgery. J Perianesthesia Nurs Off J Am Soc Perianesthesia Nurses. 2012;27(3):155-164. doi:10.1016/j.jopan.2012.02.005

38. Bergman M, Stenudd M, Engström Å. The experience of being awake during orthopaedic surgery under regional anaesthesia. Int J Orthop Trauma Nurs. 2012;16(2):88-96. doi:10.1016/j.ijotn.2011.08.004

39. Labraca NS, Castro-Sánchez AM, Matarán-Peñarrocha GA, Arroyo-Morales M, Sánchez-Joya M del M, Moreno-Lorenzo C. Benefits of starting rehabilitation within 24 hours of primary total knee arthroplasty: randomized clinical trial. Clin Rehabil. 2011;25(6):557-566. doi:10.1177/0269215510393759

40. Henderson KG, Wallis JA, Snowdon DA. Active physiotherapy interventions following total knee arthroplasty in the hospital and inpatient rehabilitation settings: a systematic review and meta-analysis. Physiotherapy. 2018;104(1):25-35. doi:10.1016/j.physio.2017.01.002

41. den Hertog A, Gliesche K, Timm J, Mühlbauer B, Zebrowski S. Pathway-controlled fast-track rehabilitation after total knee arthroplasty: a randomized prospective clinical study evaluating the recovery pattern, drug consumption, and length of stay. Arch Orthop Trauma Surg. 2012;132(8):1153-1163. doi:10.1007/s00402-012-1528-1

42. Lei Y-T, Xie J-W, Huang Q, Huang W, Pei F-X. Benefits of early ambulation within $24 \mathrm{~h}$ after total knee arthroplasty: a multicenter retrospective cohort study in China. Mil Med Res. 2021;8(1):17. doi:10.1186/s40779-021-00310-x

43. Stark ÅJ, Charalambous A, Istomina N, et al. The quality of recovery on discharge from hospital, a comparison between patients undergoing hip and knee replacement - a European study. J Clin Nurs. 2016;25(17-18):2489-2501. doi:https://doi.org/10.1111/jocn.13278

44. Lo LWT, Suh J, Chen JY, et al. Early Postoperative Pain After Total Knee Arthroplasty Is Associated With Subsequent Poorer Functional Outcomes and Lower Satisfaction. J Arthroplasty. Published online February 25, 2021. doi:10.1016/j.arth.2021.02.044

45. Specht K, Kjaersgaard-Andersen P, Pedersen BD. Patient experience in fast-track hip and knee arthroplasty - a qualitative study. J Clin Nurs. 2016;25(56):836-845. doi:10.1111/jocn.13121

46. Specht K, Agerskov H, Kjaersgaard-Andersen P, Jester R, Pedersen BD. Patients' experiences during the first 12 weeks after discharge in fast-track hip and knee arthroplasty - a qualitative study. Int J Orthop Trauma Nurs. 2018;31:13-19. doi:10.1016/j.ijotn.2018.08.002

47. Berg U, Berg M, Rolfson O, Erichsen-Andersson A. Fast-track program of elective joint replacement in hip and knee-patients' experiences of the clinical pathway and care process. J Orthop Surg. 2019;14. doi:10.1186/s13018-019-1232-8 
48. Lane JV, Hamilton DF, MacDonald DJ, Ellis C, Howie CR. Factors that shape the patient's hospital experience and satisfaction with lower limb arthroplasty: an exploratory thematic analysis. BMJ Open. 2016;6(5):e010871. doi:10.1136/bmjopen-2015-010871

49. Hoffmann JD, Kusnezov NA, Dunn JC, Zarkadis NJ, Goodman GP, Berger RA. The Shift to Same-Day Outpatient Joint Arthroplasty: A Systematic Review. J Arthroplasty. 2018;33(4):1265-1274. doi:10.1016/j.arth.2017.11.027

50. Churchill L, Pollock M, Lebedeva Y, et al. Optimizing outpatient total hip arthroplasty: perspectives of key stakeholders. Can J Surg. 2018;61(6):370-376. doi:10.1503/cjs.016117

51. Moore R, Derry S, Straube S, Ireson-Paine J, Wiffen P. Validating speed of onset as a key component of good analgesic response in acute pain. Eur J Pain Lond Engl. 2015;19(2):187-192. doi:10.1002/ejp.536

52. Høvik LH, Aglen B, Husby VS. Patient experience with early discharge after total knee arthroplasty: a focus group study. Scand J Caring Sci. 2018;32(2):833-842. doi:10.1111/scs.12514

53. Garzón-Rey JM, Arza-Valdés A, Nuevo-Gayoso M, Aguiló J. Effectiveness of patient empowerment over stress related to knee arthroplasty surgery. Enferm Clínica Engl Ed. 2018;28(3):186-193. doi:10.1016/j.enfcle.2018.05.001

54. Jones S, Alnaib M, Kokkinakis M, Wilkinson M, St Clair Gibson A, Kader D. Pre-operative patient education reduces length of stay after knee joint arthroplasty. Ann R Coll Surg Engl. 2011;93(1):71-75. doi:10.1308/003588410X12771863936765

55. Pellino T, Tluczek A, Collins M, et al. Increasing self-efficacy through empowerment: preoperative education for orthopaedic patients. Orthop Nurs. 1998;17(4):48-51, 54-59.

56. Sjøveian AKH, Leegaard M. Hip and knee arthroplasty - patient's experiences of pain and rehabilitation after discharge from hospital. Int J Orthop Trauma Nurs. 2017;27:28-35. doi:10.1016/j.ijotn.2017.07.001

57. Strickland LH, Kelly L, Hamilton TW, Murray DW, Pandit HG, Jenkinson C. Early recovery following lower limb arthroplasty: Qualitative interviews with patients undergoing elective hip and knee replacement surgery. Initial phase in the development of a patient-reported outcome measure. $J$ Clin Nurs. 2018;27(13-14):2598-2608. doi:10.1111/jocn.14086

58. McMurray J, McNeil H, Lafortune C, Black S, Prorok J, Stolee P. Measuring Patients' Experience of Rehabilitation Services Across the Care Continuum. Part II: Key Dimensions. Arch Phys Med Rehabil. 2016;97(1):121-130. doi:10.1016/j.apmr.2015.08.408

59. Dibbelt S, Schaidhammer M, Fleischer C, Greitemann B. Patient-doctor interaction in rehabilitation: The relationship between perceived interaction quality and long-term treatment results. Patient Educ Couns. 2009;76(3):328-335. doi:10.1016/j.pec.2009.07.031

60. Menkowitz B, Olivieri G, Belson O. Patient Satisfaction and Cosmetic Outcome in a Randomized, Prospective Study of Total Knee Arthroplasty Skin Closure Comparing Zip Surgical Skin Closure with Staples. Cureus. 2020;12(1). doi:10.7759/cureus.6705

61. Sundaram K, Piuzzi NS, Patterson BM, Stearns KL, Krebs VE, Mont MA. Skin closure with 2-octyl cyanoacrylate and polyester mesh after primary total knee arthroplasty offers superior cosmetic outcomes and patient satisfaction compared to staples: a prospective trial. Eur J Orthop Surg Traumatol. 2020;30(3):447-453. doi:10.1007/s00590-019-02591-4

62. B AB, J V, S RB, Khan HM. A comparative study between conventional skin sutures, staples adhesive skin glue for surgical skin closure. Int Surg J. 2019;6(3):775-782. doi:10.18203/2349-2902.isj20190489

63. Amin M, Glynn F, Timon C. Randomized trial of tissue adhesive vs staples in thyroidectomy integrating patient satisfaction and Manchester score. Otolaryngol Neck Surg. 2009;140(5):703-708. doi:10.1016/j.otohns.2009.01.003

64. Black N, Varaganum M, Hutchings A. Relationship between patient reported experience (PREMs) and patient reported outcomes (PROMs) in elective surgery. BMJ Qual Saf. 2014;23(7):534-542. doi:10.1136/bmjqs-2013-002707

65. Clement ND, Merrie KL, Weir DJ, Holland JP, Deehan DJ. Asynchronous Bilateral Total Knee Arthroplasty: Predictors of the Functional Outcome and Patient Satisfaction for the Second Knee Replacement. J Arthroplasty. 2019;34(12):2950-2956. doi:10.1016/j.arth.2019.06.056

66. Hassan E. Recall Bias can be a Threat to Retrospective and Prospective Research Designs. Internet J Epidemiol. 2005;3(2). Accessed May 12, 2020. http://ispub.com/IJE/3/2/13060

\section{Supplementary Files}

This is a list of supplementary files associated with this preprint. Click to download.

- ERASSTDMixedBMCMSKAppendixA.docx 